\title{
Very rapid effect of pitavastatin on microvascular function in comparison to rosuvastatin: reactive hyperemia peripheral arterial tonometric study
}

This article was published in the following Dove Press journal:

Drug Design, Development and Therapy

2 May 2013

Number of times this article has been viewed

\author{
Yasushi Kono' \\ Shota Fukuda' \\ Kenei Shimada ${ }^{2}$ \\ Koki Nakanishi ${ }^{2}$ \\ Kenichiro Otsuka ${ }^{2}$ \\ Tomoichiro Kubo' \\ Satoshi Jissho' \\ Haruyuki Taguchi' \\ Junichi Yoshikawa ${ }^{3}$ \\ Minoru Yoshiyama ${ }^{2}$ \\ 'Department of Medicine, Osaka \\ Ekisaikai Hospital, Nishi-ku, Osaka, \\ Japan; ${ }^{2}$ Department of Internal \\ Medicine and Cardiology, Osaka \\ City University School of Medicine, \\ Abeno-ku, Osaka, Japan; ${ }^{3}$ Nishinomiya \\ Watanabe Cardiovascular Center, \\ Nishinomiya, Japan
}

Background: It has been reported that pitavastatin improves endothelial function faster than other statins. Recently introduced reactive hyperemia peripheral arterial tonometry (RH-PAT) provides objective and quantitative assessment of peripheral microvascular function.

Purpose: This study aimed to investigate whether peripheral microvascular function improved 2 hours after pitavastatin in subjects with coronary artery disease (CAD) using RH-PAT, and the results were compared with those of rosuvastatin.

Methods: This study included 94 subjects with CAD, assigned to a group given $2 \mathrm{mg}$ of pitavastatin $(n=36)$, a group given $2.5 \mathrm{mg}$ of rosuvastatin $(n=38)$, and a control group $(n=20)$. RH-PAT examinations were performed before and 2 hours after statin administration.

Results: The RH-PAT index increased 2 hours after pitavastatin administration from $1.82 \pm 0.45$ to $2.16 \pm 0.62(P=0.02)$, whereas there were no differences in the RH-PAT index in the rosuvastatin group $(1.79 \pm 0.71$ to $1.91 \pm 0.53, P=0.09)$ and the control group $(1.68 \pm 0.36$ to $1.84 \pm 0.58, P=0.4)$. No significant changes were observed at 2 hours in serum cholesterol levels in each group.

Conclusion: The present study demonstrated that peripheral microvascular function improved 2 hours after a single clinical dose of pitavastatin, but not after rosuvastatin.

Keywords: coronary artery disease, statin, microvascular function

\section{Introduction}

Evidence from several large, randomized, controlled trials has demonstrated that the decrease in the serum cholesterol concentration produced by the 3-hydroxy-3methylglutaryl coenzyme A reductase inhibitors (statins) reduces the mortality of subjects with coronary artery disease (CAD). ${ }^{1,2}$ Previous studies have confirmed that statins have pleiotropic effects, including improvement of endothelial function, and that effects other than cholesterol reduction may contribute to reduction of cardiovascular complications. ${ }^{3}$ These putatively cholesterol-independent effects may be exerted more rapidly than cholesterol lowering itself, ranging from 1 day to several months. ${ }^{4-8}$ Previous reports have shown that pitavastatin may improve endothelial function faster than other statins. ${ }^{9,10}$ Therefore, we hypothesized that pitavastatin, but not other statins, improves microvascular function within several hours in subjects with CAD.

Reactive hyperemia peripheral arterial tonometry (RH-PAT) has recently been introduced to evaluate peripheral microvascular function. ${ }^{11-14}$ RH-PAT is designed to measure volumetric changes in the fingertip by using a probe to quantify pulse amplitude in response to reactive hyperemia. This technique has been reported to be useful in identifying cardiovascular risk factors. Considering it is noninvasive and
Correspondence: Shota Fukuda Department of Medicine, Osaka Ekisaikai Hospital, 2-I-I0 Honden, Nishi-ku, Osaka 550-0022, Japan

Tel +81665812881

Fax $+8166584 \mid 807$

Email h-syouta@mve.biglobe.ne.jp 
less operator- and observer-dependent, this method may have the potential to detect subtle differences in serial measurements of microvascular function. This study therefore aimed to investigate the very rapid effects (within 2 hours) of pitavastatin on peripheral microvascular function using RH-PAT in subjects with CAD and compare them with those of rosuvastatin.

\section{Methods}

\section{Study population}

There were 94 study subjects with hemodynamically stable CAD (68 \pm 12 years of age, 73 males) who had serum lowdensity lipoprotein (LDL)-cholesterol $\geq 100 \mathrm{mg} / \mathrm{dL}$, and/or LDL/high-density lipoprotein (HDL)-cholesterol ratio $\geq 2$. CAD was defined as $\geq 50 \%$ organic stenosis based on the classification by the American Heart Association on coronary angiography in at least one branch of the coronary artery. Subjects were excluded if they had a history of statin therapy, valvular heart disease, severe hepatic disease (history of liver cirrhosis or alanine aminotransferase $>2.5$ times upper normal), or renal failure (serum creatinine $>2.0 \mathrm{mg} / \mathrm{dL}$ ). The study subjects were assigned to the following three groups: (1) $2 \mathrm{mg}$ of pitavastatin, (2) $2.5 \mathrm{mg}$ of rosuvastatin, and (3) placebo (control group). This study was approved by the Institutional Review Board of the Osaka Ekisaikai Hospital, and all subjects gave their informed consent.

\section{Protocol}

A quiet, temperature-controlled $\left(24^{\circ} \mathrm{C}-27^{\circ} \mathrm{C}\right)$ room was used for the RH-PAT test. Subjects underwent RH-PAT examinations before and 2 hours after statin intake in the pitavastatin and rosuvastatin groups. Blood samples were taken immediately before each RH-PAT examination. These examinations were performed more than 3 hours after meals and after 30 minutes of rest lying down. In the control group, the RH-PAT tests were performed before and after 2 hours of rest without statin treatment, to investigate the effect of repeated RH-PAT tests on the results.

\section{RH-PAT examination}

RH-PAT was performed using ENDO-PAT 2000 (Itamar Medical, Caesarea, Israel). Specially designed finger probes were placed on the middle finger of each subject's hand. These probes consisted of a system of inflatable latex air cuffs connected by pneumatic tubes to an inflating device controlled through a computer algorithm. Pulsatile volume changes of the distal digit induced pressure alterations in the finger cuff, which were sensed by pressure transducers.
Subjects were instructed to remain at rest for 5 minutes to obtain a baseline measurement. After 5 minutes of baseline recording, a blood pressure cuff was inflated to $60 \mathrm{~mm} \mathrm{Hg}$ above systolic pressure or at least $200 \mathrm{~mm} \mathrm{Hg}$ in the test arm. After 5 minutes of occlusion, the cuff was rapidly deflated, with PAT tracings being recorded for a further 6 minutes. The ratio of the PAT signal after cuff deflation compared with baseline was calculated though a computer algorithm automatically normalizing for the baseline signal and indexed to the contralateral arm.

\section{Statistical analysis}

Values are expressed as mean \pm standard deviation. Comparisons of the RH-PAT index and laboratory and hemodynamic data before and after statin administration were performed using the Mann-Whitney U test. The chi-square test was used for comparison of categorical variables. One-way analysis of variance followed by a post-hoc Bonferroni test was used to compare the three groups (pitavastatin, rosuvastatin, and control groups). Differences were considered significant at $P<0.05$.

Inter- and intra-observer variabilities of RH-PAT examination were examined in 10 healthy subjects ( 8 males, mean age $31 \pm 5$ years) who had no history of cardiac disease or risk factors. Inter-observer variability for RH-PAT examination was analyzed with two independent blinded observers. Intra-observer variability was analyzed with the same observer at two different time points. The results were analyzed by both least squares fit linear regression analysis and the Bland-Altman method.

\section{Results}

Of the 94 subjects, 36 subjects were assigned to the pitavastatin group, 38 subjects to the rosuvastatin group, and 20 subjects to the control group. The clinical characteristics of the three groups are summarized in Table 1. There were no correlations between baseline RH-PAT index and medications, including beta-blockers, calcium channel blockers, angiotensin converting enzyme inhibitors or angiotensin receptor blockers, and aspirin, as shown in Figure 1. There was no significant difference in the baseline value of the RH-PAT index among the three groups: $1.82 \pm 0.45$ in the pitavastatin group, $1.79 \pm 0.71$ in the rosuvastatin group, and $1.68 \pm 0.36$ in the controls $(P=0.6)$. Further, the baseline laboratory parameters and hemodynamics did not differ significantly among the three groups (Figure 2).

The RH-PAT index increased significantly 2 hours after pitavastatin administration, from $1.82 \pm 0.45$ to $2.16 \pm 0.62$ $(P=0.02)$, as shown in Figure 2. However, there were no 
Table I Clinical characteristics of the pitavastatin, rosuvastatin, and control groups

\begin{tabular}{|c|c|c|c|c|}
\hline & $\begin{array}{l}\text { Pitavastatin } \\
(n=36)\end{array}$ & $\begin{array}{l}\text { Rosuvastatin } \\
(\mathrm{n}=\mathbf{3 8})\end{array}$ & $\begin{array}{l}\text { Control } \\
(n=20)\end{array}$ & $P$-value \\
\hline Age, years & $68 \pm 12$ & $63 \pm 12$ & $70 \pm 11$ & 0.08 \\
\hline Sex (male), n (\%) & $30(83 \%)$ & $29(76 \%)$ & 14 (70\%) & 0.5 \\
\hline Hypertension, n (\%) & 27 (75\%) & 31 (82\%) & 17 (85\%) & 0.6 \\
\hline Hyperlipidemia, n (\%) & $19(53 \%)$ & $20(53 \%)$ & 7 (35\%) & 0.4 \\
\hline Diabetes, n (\%) & $17(47 \%)$ & I8 (47\%) & $8(40 \%)$ & 0.8 \\
\hline Body mass index, $\mathrm{kg} / \mathrm{m}^{2}$ & $23.6 \pm 3.9$ & $23.2 \pm 4.1$ & $22.8 \pm 4.6$ & 0.5 \\
\hline Previous history of MI, n (\%) & $8(22 \%)$ & $8(22 \%)$ & $5(25 \%)$ & 0.9 \\
\hline Previous history of coronary & $20(56 \%)$ & 15 (39\%) & II (55\%) & 0.3 \\
\hline \multicolumn{5}{|l|}{ revascularization, n (\%) } \\
\hline \multicolumn{5}{|l|}{ Medication } \\
\hline Beta-blockers, n (\%) & $10(28 \%)$ & $9(24 \%)$ & $5(25 \%)$ & 0.9 \\
\hline Calcium channel blockers, n (\%) & $20(56 \%)$ & $13(34 \%)$ & $8(40 \%)$ & 0.2 \\
\hline ACEI or ARB, $n(\%)$ & $22(61 \%)$ & $28(74 \%)$ & $12(60 \%)$ & 0.4 \\
\hline Aspirin, n (\%) & $24(67 \%)$ & $22(58 \%)$ & II (55\%) & 0.6 \\
\hline
\end{tabular}

Note: Values are mean \pm SD.

Abbreviations: ACEl, angiotensin converting enzyme inhibitor; ARB, angiotensin receptor blockers; MI, myocardial infarction.

differences in the RH-PAT index in the rosuvastatin group $(1.79 \pm 0.71$ to $1.91 \pm 0.53, P=0.09)$ and the control group (1.68 \pm 0.36 to $1.84 \pm 0.58, P=0.4)$. Moreover, there were no significant differences in any laboratory parameters before and after statin intake in each group.

Excellent correlations were observed for inter-observer and intra-observer variabilities of RH-PAT examination. Values were $r=0.92$ and $r=0.95$ for the RH-PAT index. From the Bland-Altman method, inter- and intra-observer variabilities for the RH-PAT index were 0.04 and 0.06 , respectively.

\section{Discussion}

\section{Advantages of RH-PAT over flow-mediated dilation (FMD)}

FMD of the brachial artery is a widely used method for the study of endothelial function in clinical practice. ${ }^{15-17}$

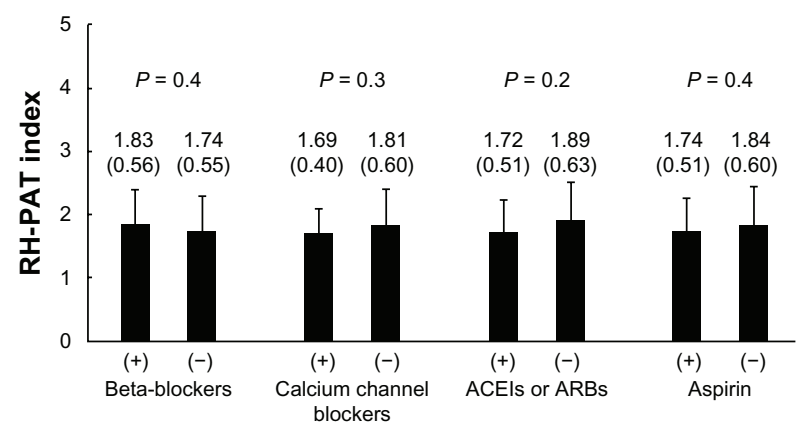

Figure I Baseline RH-PAT index and medications.

Notes: Values are expressed as mean (standard deviation). P-values showed the results of the comparison of RH-PAT index before and after treatment in each medication.

Abbreviations: ACEl, angiotensin converting enzyme inhibitor; ARB, angiotensin receptor blocker; RH-PAT, reactive hyperemia peripheral arterial tonometry.
However, this method requires a certain degree of skill to obtain accurate measurements of brachial artery diameter. Furthermore, sympathetic nervous activity and subsequent hemodynamic changes may affect the FMD result. ${ }^{15,18}$ The RH-PAT system has the potential to overcome these shortcomings of the FMD test. This method is an automatic, less operator-dependent, quantitative test for digital volume measurement of the hyperemic response, and by normalizing using the contralateral arm measurement, the effect of hemodynamic changes can be eliminated. ${ }^{11-14}$ Importantly, the relationship between or a comparison of the FMD and RH-PAT index needs careful consideration. Some studies have indicated that FMD and RH-PAT index reflect different pathologies of peripheral vascular endothelial function. ${ }^{19,20}$ FMD is used to assess large artery reactivity, whereas the RH-PAT index reflects microvascular function. The RHPAT index has been shown to be at least $50 \%$ dependent on endothelial nitric oxide (NO) activity. ${ }^{21}$ Therefore, the RHPAT index may be suitable for assessing subtle differences in serial measurements of microvascular function.

\section{Rapid effects of statins on endothelial function}

Previous clinical investigations have demonstrated that the effect of statins on peripheral and coronary endothelial function occurs within 24 hours to several months. O'Driscoll et al demonstrated that 4-week simvastatin treatment improved FMD in subjects with dyslipidemia. ${ }^{5}$ A similar positive effect on microvascular function was recently confirmed by Nonogaki et al using RH-PAT 4 weeks after pitavastatin therapy. ${ }^{6}$ Tsunekawa et al reported that cerivastatin improved 

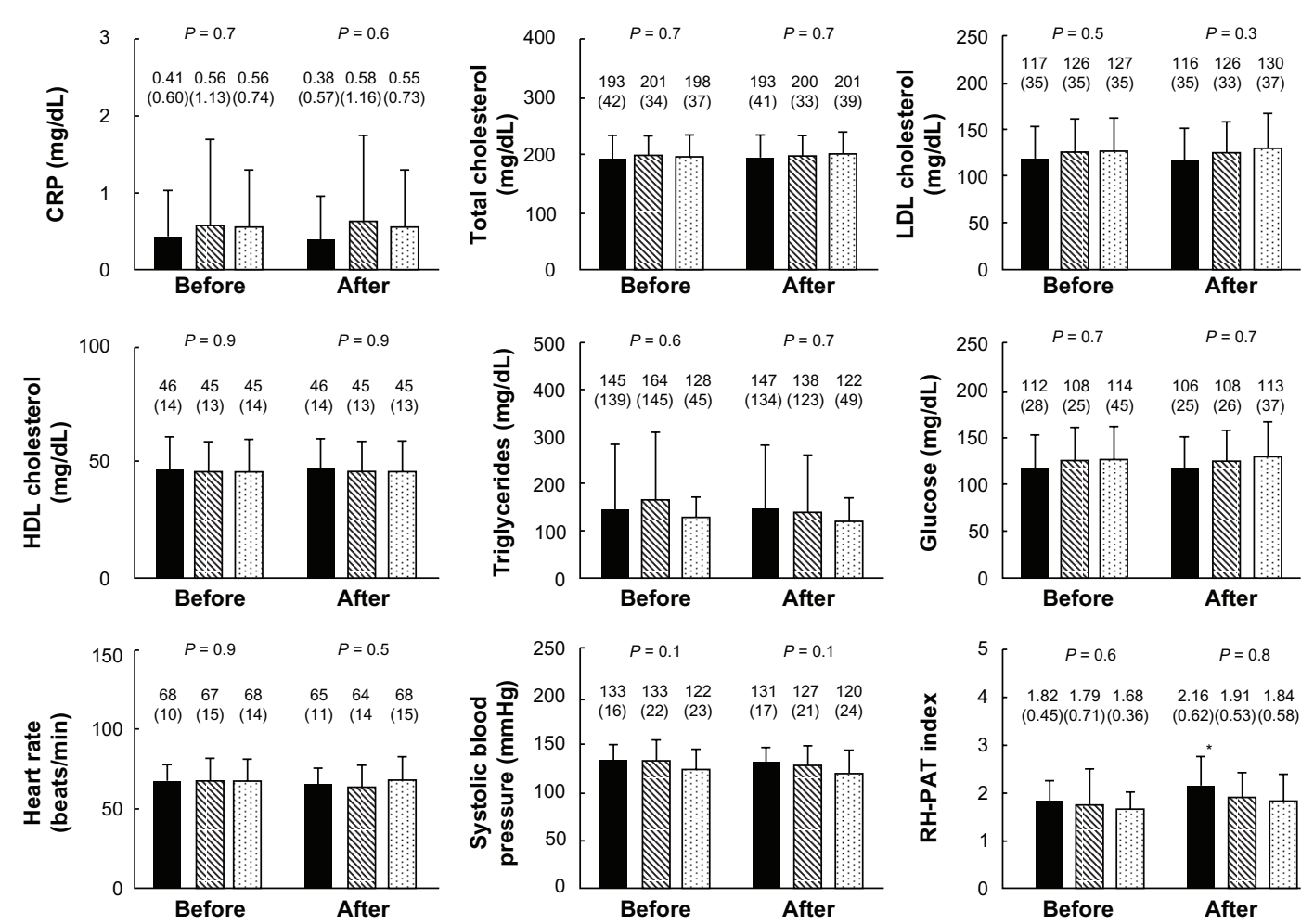

Figure 2 RH-PAT index before and after treatment in the pitavastatin group (left), rosuvastatin group (middle), and control group (right).

Notes: Values are expressed as means (standard deviation). $P$-values represent the results of one-way analysis of variance before and after treatment, respectively. $*$ denotes $P<0.05$ versus the corresponding parameter before treatment.

Abbreviations: CRP, C-reactive protein; HDL, high-density lipoprotein; LDL, low-density lipoprotein; RH-PAT, reactive hyperemia peripheral arterial tonometry.

FMD within 3 days in subjects with diabetes, independent of its lipid lowering effect. ${ }^{7}$ Wassmann et al, who measured coronary flow reserve, also showed that endothelial-dependent coronary vasomotion increased 24 hours after a single treatment of pravastatin in subjects with CAD. ${ }^{8}$ The present study demonstrated that pitavastatin improved microvascular function rapidly, within 2 hours. This finding may indicate the importance of early and new therapeutic approaches with pitavastatin with respect to improved endothelial function contributing to improved cardiovascular outcomes, such as in the settings of acute coronary syndrome and heart failure.

\section{Potential mechanisms of the difference between pitavastatin and rosuvastatin}

The advantage of pitavastatin over rosuvastatin in the rapid improvement of peripheral microvascular function was demonstrated in the present study, as in previous studies. Sakabe et al examined FMD tests 2 and 12 weeks after pitavastatin and atorvastatin, respectively. ${ }^{9}$ They found that the short-term effect of pitavastatin was superior to that of atorvastatin. An intravascular ultrasonographic study also reported that the positive effect of pitavastatin on coronary plaques was faster than that seen with atorvastatin. ${ }^{10}$
These results might be explained as follows. First, pitavastatin administered orally reaches a peak plasma concentration $\left(\mathrm{C}_{\max }\right)$ in approximately 1 hour. The $\mathrm{C}_{\max }$ at a dose of $2 \mathrm{mg}$ pitavastatin is $26.1 \mathrm{ng} / \mathrm{mL}$, which is higher than that of $5 \mathrm{mg}$ rosuvastatin $(3.6 \mathrm{ng} / \mathrm{mL}) .{ }^{22,23}$ Faster transfer of the drug to blood vessels may result in increased bioavailability of $\mathrm{NO}$ through the inhibition of Rho-kinase in endothelial cells. An experimental study showed that Rho-kinase inhibition rapidly activated Akt/PI3 kinase within 15 to 30 minutes and increased in a concentration-dependent manner. ${ }^{24}$ Second, the pleiotropic effects of statins may depend on their lipophilicity. Because the cell membrane consists of lipid bilayers, intracellular pathways and possible effects of statins differ between lipophilic and hydrophilic statins. It has been reported that cerivastatin, a lipophilic statin, was more effective with the respect to endothelial NO synthase expression for 12 hours than rosuvastatin, a hydrophilic statin, in subjects with diabetes. ${ }^{25}$ Furthermore, short-term cardiovascular outcomes after acute myocardial infarction were better in subjects with a lipophilic statin than with a hydrophilic statin. ${ }^{26}$ Finally, pitavastatin may simply have a stronger effect on endothelial function than other statins. Also, pitavastatin is marginally metabolized by cytochrome 
P450 enzymes, and is less dependent on organic aniontransporting polypeptides for its uptake into hepatocytes. ${ }^{27,28}$ Such a unique pharmacological profile of pitavastatin might have novel and wide-ranging effects on endothelial function. The present study demonstrated that pitavastatin resulted in rapid improvement of microvascular function. Since endothelial dysfunction is strongly associated with the onset and development of CAD and considerable mortality despite contemporary therapies, ${ }^{16,29,30}$ early modification of endothelial function is of crucial importance. The management of $\mathrm{CAD}$ in clinical practice could be improved by considering the physicochemical characteristics of statins. Also, a distinctive metabolic pathway of pitavastatin is associated with a favorable drug-drug interaction profile; this supports the use of pitavastatin among the statins currently available. ${ }^{28,31}$

\section{Study limitations}

Several limitations need to be considered. First, the study was initially designed to include 60 subjects ( 20 subjects in each group). However, because of wider distribution of the RH-PAT index in the rosuvastatin group than in the other groups, 36 subjects were added, randomly assigned to pitavastatin or rosuvastatin groups on the basis of the value of first RH-PAT index. Two subjects with pitavastatin then refused to perform second RH-PAT test due to pain of cuff-inflation. Crossover designed studies with a larger number of subjects would be ideal to assess the acute effect of two different drugs. Another ideal way would be to randomize subjects to the different treatment groups to balance out other factors. Further, although observer variability of RH-PAT values was small, the results of a one-way analysis of variance did not account for observer variability. Second, this study included subjects with CAD, and the application of these results to other populations may be limited. Furthermore, 73 (78\%) subjects continued taking one or more antihypertensive drugs during the examinations, such as angiotensin-converting enzyme inhibitors and angiotensin receptor blockers, which might have altered endothelial function and affected the results of this study. ${ }^{32,33}$ Although no correlations were observed between baseline RH-PAT index and medications, the time of intake of these medications might have altered endothelial function and thus affected the results of this study. Third, RH-PAT index was slightly increased even in the control group, which may be due to 2-hour rest and/or circadian variation of endothelial function. ${ }^{34}$ The first examination was performed in the morning in all subjects, and this question may be resolved if the subjects were randomly assigned with respect to the time of day when the first study was done. Finally, a dose of $2.5 \mathrm{mg}$ of rosuvastatin was used because this is the recommended starting dose in Japan. It remains to be seen whether different doses will show results similar to those found in this study. The very rapid increase in the RH-PAT index after pitavastatin administration may be associated with a decrease in oxidative stress, which inactivates NO. Measurement of plasma concentrations of oxidative stress markers, thiobarbituric acid reactive substances, isoprostanes, and 8-hydroxydeoxyguanosine may illuminate the presumed underlying mechanisms of this finding, and nitroglycerin-mediated vasodilation, as a marker of endothelium-independent vasodilation, should be measured in a future study.

\section{Conclusion}

This RH-PAT study demonstrated that single clinical doses of pitavastatin, but not of rosuvastatin, improved peripheral microvascular function within 2 hours in subjects with CAD. These results may support early intervention of statin therapy in the management of CAD and other atherosclerotic diseases.

\section{Acknowledgments}

This work was supported in part by a research grant from the Osaka Foundation for the Prevention of Cancer and Cardiovascular Diseases.

\section{Disclosure}

The authors report no conflicts of interest in this work.

\section{References}

1. Randomised trial of cholesterol lowering in 4444 patients with coronary heart disease: the Scandinavian Simvastatin Survival Study (4S). Lancet. 1994;344(8934):1383-1389.

2. Shepherd J, Cobbe SM, Ford I, et al. Prevention of coronary heart disease with pravastatin in men with hypercholesterolemia. West of Scotland Coronary Prevention Study Group. $N$ Engl J Med. 1995;333(20):1301-1307.

3. Kirmizis D, Papagianni A, Dogrammatzi F, et al. Effects of simvastatin on markers of inflammation, oxidative stress and endothelial cell apoptosis in patients on chronic hemodialysis. J Atheroscler Thromb. 2010;17(12):1256-1265.

4. Takemoto M, Liao JK. Pleiotropic effects of 3-hydroxy-3-methylglutaryl coenzyme a reductase inhibitors. Arterioscler Thromb Vasc Biol. 2001;21(11):1712-1719.

5. O'Driscoll G, Green D, Taylor RR. Simvastatin, an HMG-coenzyme A reductase inhibitor, improves endothelial function within 1 month. Circulation. 1997;95(5):1126-1131.

6. Nonogaki K, Suzuki M, Kanai N, Sumii M, Kaji T. Short-term effect of pitavastatin on the reactive hyperemic index in post-menopausal women with high levels in serum LDL-cholesterol. Int J Cardiol. 2011;150(2):227-228.

7. Tsunekawa T, Hayashi T, Kano H, et al. Cerivastatin, a hydroxymethylglutaryl coenzyme a reductase inhibitor, improves endothelial function in elderly diabetic patients within 3 days. Circulation. 2001;104(4):376-379. 
8. Wassmann S, Faul A, Hennen B, Scheller B, Bohm M, Nickenig G. Rapid effect of 3-hydroxy-3-methylglutaryl coenzyme a reductase inhibition on coronary endothelial function. Circ Res. 2003;93(9):e98-e103.

9. Sakabe K, Fukuda N, Fukuda Y, et al. Comparisons of short- and intermediate-term effects of pitavastatin versus atorvastatin on lipid profiles, fibrinolytic parameter, and endothelial function. Int J Cardiol. 2008;125(1):136-138

10. Toi T, Taguchi I, Yoneda S, et al. Early effect of lipid-lowering therapy with pitavastatin on regression of coronary atherosclerotic plaque. Comparison with atorvastatin. Circ J. 2009;73(8):1466-1472.

11. Bonetti PO, Barsness GW, Keelan PC, et al. Enhanced external counterpulsation improves endothelial function in patients with symptomatic coronary artery disease. J Am Coll Cardiol. 2003;41(10):1761-1768.

12. Hamburg NM, Keyes MJ, Larson MG, et al. Cross-sectional relations of digital vascular function to cardiovascular risk factors in the Framingham Heart Study. Circulation. 2008;117(19):2467-2474.

13. Matsuzawa Y, Sugiyama S, Sugamura K, et al. Digital assessment of endothelial function and ischemic heart disease in women. $J \mathrm{Am}$ Coll Cardiol. 2010;55(16):1688-1696.

14. Rubinshtein R, Kuvin JT, Soffler M, et al. Assessment of endothelial function by non-invasive peripheral arterial tonometry predicts late cardiovascular adverse events. Eur Heart J. 2010;31(9):1142-1148.

15. Corretti MC, Anderson TJ, Benjamin EJ, et al. Guidelines for the ultrasound assessment of endothelial-dependent flow-mediated vasodilation of the brachial artery: a report of the International Brachial Artery Reactivity Task Force. J Am Coll Cardiol. 2002;39(2):257-265.

16. Gokce N, Keaney JF Jr, Hunter LM, et al. Predictive value of noninvasively determined endothelial dysfunction for long-term cardiovascular events in patients with peripheral vascular disease. J Am Coll Cardiol. 2003;41(10):1769-1775.

17. Shimada K, Fukuda S, Maeda K, et al. Aromatherapy alleviates endothelial dysfunction of medical staff after night-shift work: preliminary observations. Hypertens Res. 2011;34(2):264-267.

18. Hijmering ML, Stroes ES, Pasterkamp G, Sierevogel M, Banga JD, Rabelink TJ. Variability of flow mediated dilation: consequences for clinical application. Atherosclerosis. 2001;157(2):369-373.

19. Hamburg NM, Palmisano J, Larson MG, et al. Relation of brachial and digital measures of vascular function in the community: the Framingham heart study. Hypertension. 2011;57(3):390-396.

20. Schnabel RB, Schulz A, Wild PS, et al. Noninvasive vascular function measurement in the community: cross-sectional relations and comparison of methods. Circ Cardiovasc Imaging. 2011;4(4):371-380.
21. Nohria A, Gerhard-Herman M, Creager MA, Hurley S, Mitra D, Ganz P. Role of nitric oxide in the regulation of digital pulse volume amplitude in humans. J Appl Physiol. 2006;101(2):545-548.

22. Livalo $^{\circledR}$ (pitavastatin) [prescribing information]. Tokyo, Japan: Kowa Company, Ltd; 2010.

23. Crestor $^{\circledR}$ (rosuvastatin). [prescribing information]. Osaka, Japan: AstraZeneca; 2011.

24. Wolfrum S, Dendorfer A, Rikitake Y, et al. Inhibition of Rho-kinase leads to rapid activation of phosphatidylinositol 3-kinase/protein kinase Akt and cardiovascular protection. Arterioscler Thromb Vasc Biol. 2004;24(10):1842-1847.

25. Jantzen F, Konemann S, Wolff B, et al. Isoprenoid depletion by statins antagonizes cytokine-induced down-regulation of endothelial nitric oxide expression and increases NO synthase activity in human umbilical vein endothelial cells. J Physiol Pharmacol. 2007;58(3):503-514.

26. Kim MC, Ahn Y, Jang SY, et al. Comparison of clinical outcomes of hydrophilic and lipophilic statins in patients with acute myocardial infarction. Korean J Intern Med. 2011;26(3):294-303.

27. Davignon J. Pleiotropic effects of pitavastatin. Br J Clin Pharmacol. 2012;73(4):518-535.

28. Catapano AL. Statin-induced myotoxicity: pharmacokinetic differences among statins and the risk of rhabdomyolysis, with particular reference to pitavastatin. Curr Vasc Pharmacol. 2012;10(2):257-267.

29. Kitta Y, Obata JE, Nakamura T, et al. Persistent impairment of endothelial vasomotor function has a negative impact on outcome in patients with coronary artery disease. J Am Coll Cardiol. 2009;53(4):323-330.

30. Yoshino S, Hamasaki S, Ishida S, et al. Relationship between bilirubin concentration, coronary endothelial function, and inflammatory stress in overweight patients. J Atheroscler Thromb. 2011;18(5):403-412.

31. Corsini A, Ceska R. Drug-drug interactions with statins: will pitavastatin overcome the statins' Achilles' heel? Curr Med Res Opin. 2011;27(8):1551-1562.

32. Ghiadoni L, Magagna A, Versari D, Kardasz I, Huang Y, Taddei S, Salvetti A. Different effect of antihypertensive drugs on conduit artery endothelial function. Hypertension. 2003;41(6):1281-1286.

33. Wassmann S, Hilgers S, Laufs U, Bohm M, Nickenig G. Angiotensin II type 1 receptor antagonism improves hypercholesterolemiaassociated endothelial dysfunction. Arterioscler Thromb Vasc Biol. 2002;22(7):1208-1212.

34. Fukuda S, Shimada K, Maeda K, et al. Circadian variation in coronary flow velocity reserve and its relation to alpha1-sympathetic activity in humans. Int J Cardiol. 2012;157(2):216-220.
Drug Design, Development and Therapy

\section{Publish your work in this journal}

Drug Design, Development and Therapy is an international, peerreviewed open-access journal that spans the spectrum of drug design and development through to clinical applications. Clinical outcomes, patient safety, and programs for the development and effective, safe, and sustained use of medicines are a feature of the journal, which

\section{Dovepress}

has also been accepted for indexing on PubMed Central. The manuscript management system is completely online and includes a very quick and fair peer-review system, which is all easy to use. Visit http://www.dovepress.com/testimonials.php to read real quotes from published authors. 